\title{
High resolution gamma-ray spectroscopy of fast-neutron induced fission
}

\author{
Jonathan Wilson ${ }^{1, *}$, Damien Thisse ${ }^{1}$, Matthieu Lebois ${ }^{1}$, Nikola Jovancevic $^{1}$, \\ Rhiann Canavan ${ }^{2,3}$, Mathias Rudigier ${ }^{2,4}$, David Étasse ${ }^{5}$, and Rosa-Belle Gerst ${ }^{6}$ \\ ${ }^{1}$ Université Paris-Saclay, CNRS/IN2P3, IJCLab, 91405 Orsay, France \\ ${ }^{2}$ Department of Physics, University of Surrey, Guildford GU2 7XH, UK \\ ${ }^{3}$ National Physical Laboratory, Hampton Road, Teddington, Middlesex TW11 0LW, UK \\ ${ }^{4}$ Technische Universität Darmstadt, Fachbereich Physik, Institut für Kernphysik, 64289 Darmstadt, \\ Germany \\ ${ }^{5}$ LPC Caen, 6 Boulevard Maréchal Juin, 14000 Caen, France \\ ${ }^{6}$ Institut für Kernphysik, Universität zu Köln, 50937 Köln, Germany
}

\begin{abstract}
Experiments to perform precision spectroscopy of fast neutron induced fission were carried out during the $v$-Ball experimental campaign at the ALTO facility of IJC Laboratory Orsay. Low energy fission of ${ }^{232} \mathrm{Th}(\mathrm{n}, \mathrm{f})$, ${ }^{238} \mathrm{U}(\mathrm{n}, \mathrm{f})$ and spontaneous fission of ${ }^{252} \mathrm{Cf}$ were studied using this hybrid highresolution spectrometer and calorimeter. New observables such as $\gamma$-ray multiplicity distributions correlated with specific fission fragments are presented and discussed. A new method using fast-timing techniques to detect prompt fission neutrons in coincidence with prompt fission $\gamma$-rays is described.
\end{abstract}

\section{Introduction}

The $v$-Ball experimental campaign was carried out in 2018 at the ALTO facility of the IJC Laboratory in Orsay. The $v$-Ball spectrometer [1] is a hybrid Germanium, BGO and $\mathrm{LaBr}_{3}$ spectrometer ideally suited to the study of fast-neutron induced fission.

The unique features of this device are as follows: (i) a hybrid spectrometer combining the high energy resolution of HPGe detectors with the excellent time resolution of $\mathrm{LaBr}_{3}$ detectors; (ii) a fully digital acquisition system capable of running in triggered or triggerless modes; (iii) a high $\gamma$-ray detection efficiency; (iv) the capacity to perform $\gamma$-ray calorimetry due to the large solid angle coverage and (v) the ability to couple the spectrometer to the LICORNE directional neutron source [2], [3] to perform spectroscopy of neutron-induced reactions. The total Germanium photopeak efficiency was $6.7 \%$ at $1 \mathrm{MeV}$, and the total calorimeter photopeak efficiency was estimated at $48 \%$.

The experimental campaign for fission studies took place over a period of 7 weeks to investigate fast neutron induced fission of ${ }^{232} \mathrm{Th}(\mathrm{n}, \mathrm{f})$ at an average neutron energy of $1.7 \mathrm{MeV}$, ${ }^{238} \mathrm{U}(\mathrm{n}, \mathrm{f})$ at average neutron energies of 1.7 and $3.5 \mathrm{MeV}$, and spontaneous fission of ${ }^{252} \mathrm{Cf}$. The twin goals of these experiments were nuclear structure studies via high-resolution spectroscopy of neutron rich isotopes and studies of the fission process itself. The latter goal is the focus of this current paper.

\footnotetext{
*e-mail: jonathan.wilson@ijclab.in2p3.fr
} 


\subsection{Correlated fission observables}

The fission observables accessible during the experiments were as follows:

- Prompt and delayed $\gamma$-ray multiplicity distributions

- Prompt and delayed $\gamma$-ray sum energy distributions

- Average prompt $\gamma$-ray multiplicities and total energies, $<\mathrm{M} \gamma>$ and $<E_{\mathrm{tot}}>$

- High energy $\gamma$-ray spectra up to $10 \mathrm{MeV}$

- Average prompt neutron multiplicities from fast timing (see Sect. 2)

which could all be correlated with the detection of individual fragments from observation of the characteristic prompt and delayed $\gamma$-ray decays in the HPGe detectors.

\section{Prompt $\gamma$-ray multiplicity distributions}

Prompt $\gamma$-ray multiplicity distributions are spin-sensitive observables that may contain interesting information which should be reproducible be fission models. These distributions correlated with individual fission fragments can be extracted by double-gating on $\gamma$-ray transitions in the HPGe detectors. Each multiplicity distribution will always have equal contributions from the nucleus in question and its binary partner fragments. It is not possible to separate the partial multiplicity contributions the two sources.

Currently only relative differences between these distributions are compared. To obtain absolute multiplicity distributions requires the application of a complicated unfolding procedure with the simulated $v$-Ball response to $\gamma$-rays to events of different multiplicity and sum energies. The $v$-Ball calorimeter is $68 \%$ efficient at detecting $\gamma$ rays of $1 \mathrm{MeV}$. To obtain estimated absolute values for average multiplicities a scale factor of 1.3 can be applied.

\subsection{Multiplicity distributions gating on spherical nuclei}

The doubly magic nucleus ${ }^{132} \mathrm{Sn}$ is a special case when produced in fission, since it has a spherical shape and is very hard to deform. Its excitation spectrum is that of a vibrator rather than a rotor. In our previous work a large anomaly in the fission yield for this nucleus and its near neighbours was observed using measurement techniques of $\gamma$-ray spectroscopy [4]. Yields 500\%-600\% lower than expected were observed in the ${ }^{238} \mathrm{U}(\mathrm{n}, \mathrm{f})$ reaction when compared to the available evaluations which are based on theory/extrapolations from other fissioning systems.

One explanation proposed for the origin of this anomaly was the low average $\gamma$-ray multiplicity, which would be theoretically emitted from ${ }^{132} \mathrm{Sn}$ due to its spherical shape at scission [5]. A lower multiplicity distribution associated with detection of this nucleus might cause a significant trigger bias and subsequent underestimation of its yield.

Figure 1 shows the coincident multiplicity distributions from the recent $v$-Ball experiments for ${ }^{132} \mathrm{Sn}$ and its $3 \mathrm{n}$ partner nucleus ${ }^{104} \mathrm{Mo}$. The shape of these distributions is observed to be very similar to that of other isotopes with a long tail extending to high multiplicities ( $>$ 10). This suggests that the explanation given in [5] cannot be the origin of the yield anomaly. Since ${ }^{132} \mathrm{Sn}$ is always emitted with a Mo partner nucleus, it is possible that a deficit of $\gamma$-rays from ${ }^{132} \mathrm{Sn}$ are compensated by a surplus of $\gamma$-rays from the partner.

Subsequent experimental work on fission yields of ${ }^{238} \mathrm{U}(\mathrm{n}, \mathrm{f})$ also using the $\gamma$-ray spectroscopy technique by Fotiades et al. [6] shows that experimentally deduced normalized 
yields for $\mathrm{Sn}$ isotopes 3 times larger if only single $\gamma$ rays are used to identify the fragments, instead of $\gamma-\gamma$ coincidences (used in [4]). A more recent experiment by Ramos et al. [7] measured yields for ${ }^{238} \mathrm{U}(\mathrm{n}, \mathrm{f})$ using the surrogate ${ }^{9} \mathrm{Be}\left({ }^{238} \mathrm{U},{ }^{239} \mathrm{U}^{*}\right){ }^{8} \mathrm{Be}$ reaction which allows the possibility for direct identification of the fragments due to the inverse kinematics. Here, normalized fission yields were observed to be double those measured in [6], although it is not clear what the impact of the significant angular momentum mismatch between the surrogate and neutron-induced reactions had on the results. The remaining difference could possibility be due to a significant $0+$ feeding, which was unobservable in [6].

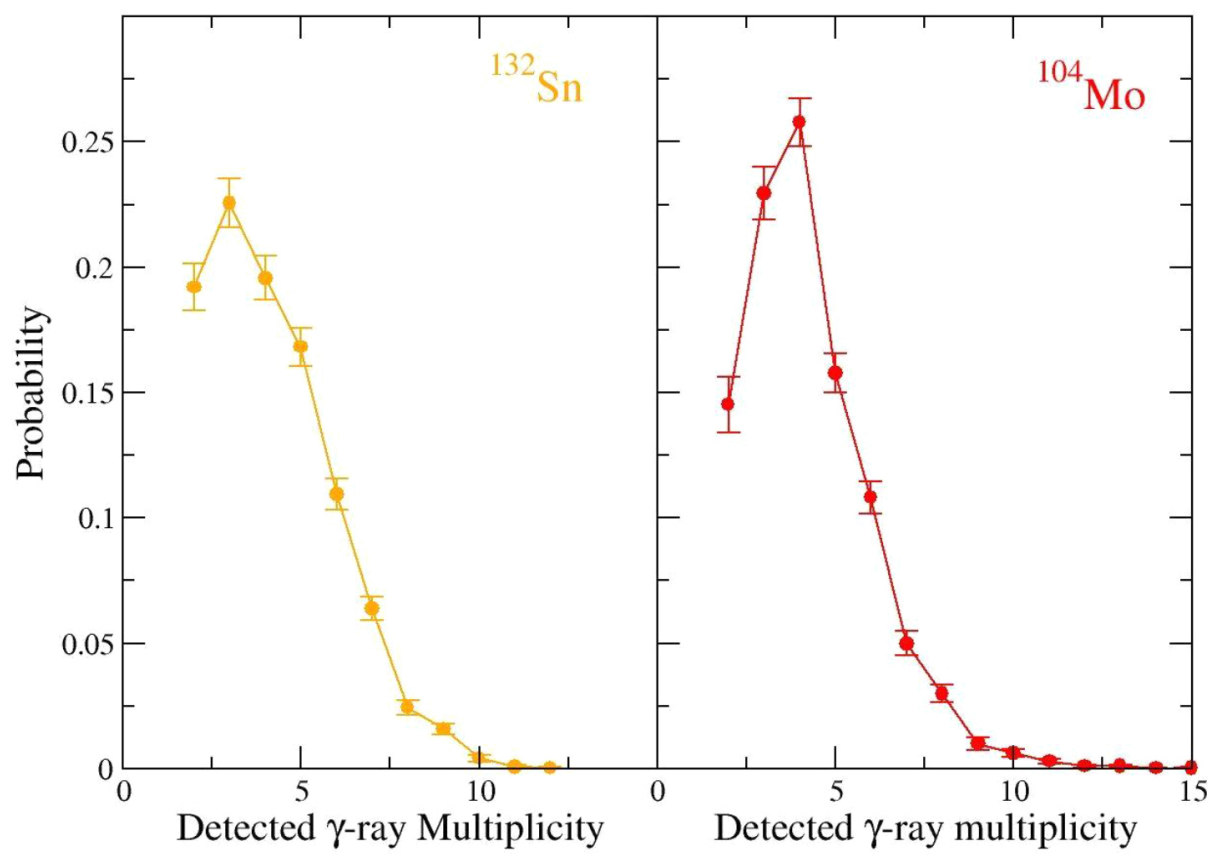

Figure 1. Comparison of the detected $\gamma$-ray multiplicity distributions for ${ }^{132} \mathrm{Sn}$ and ${ }^{104} \mathrm{Mo}$ from the ${ }^{238} \mathrm{U}(\mathrm{n}, \mathrm{f})$ data. Fragments are identified by double-gating on the $4^{+} \longrightarrow 2^{+}$and $2^{+} \longrightarrow 0^{+}$transitions in the HPGe detectors.

A significant part of the fission yield anomaly is clearly due to the explanation suggested by Fotiades et al., that for ${ }^{132} \mathrm{Sn}$ and its neighbours there will be a large direct side-feeding of the $2+$ states. A lowering of the measured intensity occurs when using $\gamma-\gamma$ coincidence spectroscopy to detect the isotope since the $2+$ side-feeding is missed, but the effect is eliminated by using single $\gamma$ ray detection of the $2+^{+} \longrightarrow 0^{+}$transitions. This explanation is confirmed by our current data, where direct side-feedings can now be measured by gating on the partner nucleus and measuring $4^{+} \longrightarrow 2^{+}$and $2^{+} \longrightarrow 0^{+}$ratios in the spectrum. The direct side-feedings are large and found to be large (up to $60 \%$ ) in the case of ${ }^{132} \mathrm{Sn}$ and neighbours, while small for other nuclei.

\subsection{Average multiplicity dependence on fragment mass}

The evolution of average prompt $\gamma$-ray multiplicities as a function of the mass of the fragments may give interesting information, which ought to be reproducible by fission models. 
In Fig. 2, we show the average multiplicity values deduced from double-gating on many different fragments produced in the ${ }^{252} \mathrm{Cf}$ reaction. Here, fission events were tagged directly by detection in an ionisation chamber placed in the centre of the array [8]. One fragment is stopped, while the other is detected in flight. The results show that the average multiplicity values are remarkably similar with very little variation as a function of mass. Fits to the data of two quadratic function in the light and heavy peaks show are clearly symmetric with a slight convex curvature towards fragments at peak yields, where average fragment multiplicity is $\sim 10 \%$ higher. The average multiplicities always have two equal components from both the light and heavy peak partners, which are not possible to separate. Hence no saw-tooth patterns [9], [10] are evident due to high $\gamma$-ray multiplicity in one fragment summing with low multiplicity in the other fragment an vice-versa, resulting in a total multiplicity which is fairly constant.

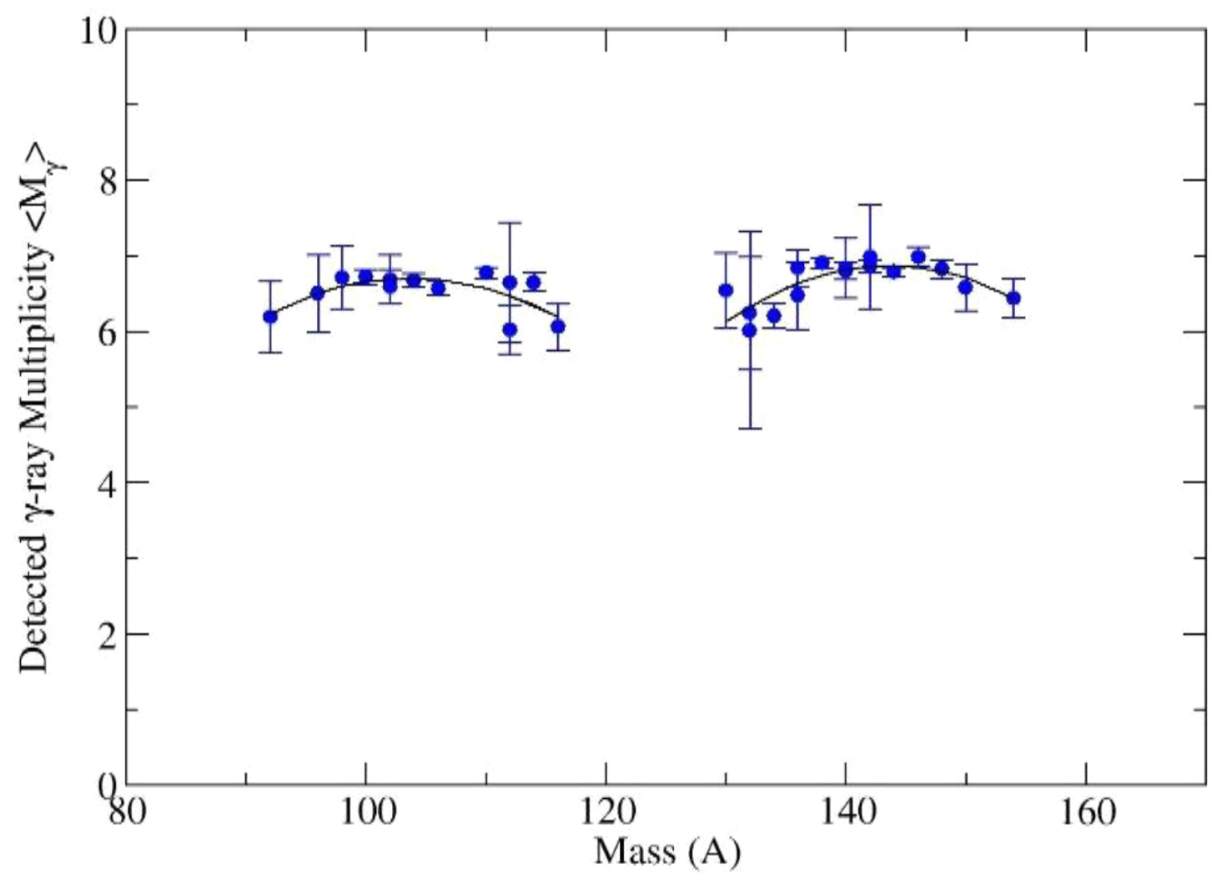

Figure 2. Average detected prompt $\gamma$-ray multiplicities for a range of even-even fragments produced in the ${ }^{252} \mathrm{Cf}(\mathrm{SF})$ reaction. Each fragment is identified by double gating on its $4^{+} \longrightarrow 2^{+}$and $2^{+} \longrightarrow 0^{+}$ transitions in the HPGe detectors. Quadratic fits to the experimental data in heavy and light peaks are shown with the black lines.

\subsection{Comparison of multiplicity distributions between the three systems}

Multiplicity distributions can also be compared across all three of the fissioning systems. Figure 3 shows an example of the distributions for the ${ }^{144} \mathrm{Ba}$ nucleus which is close to the centre of the heavy peak for all three systems. The most frequently produced partner nuclei of ${ }^{144} \mathrm{Ba}$ are ${ }^{104} \mathrm{Mo},{ }^{92} \mathrm{Kr}$ and ${ }^{86} \mathrm{Se}$ for ${ }^{252} \mathrm{Cf}(\mathrm{SF}),{ }^{238} \mathrm{U}(\mathrm{n}, \mathrm{f})$ and ${ }^{232} \mathrm{Th}(\mathrm{n}, \mathrm{f})$ respectively. Large differences in multiplicity can be observed indicating a large drop in multiplicity as the fissioning system becomes lighter. 


\section{Correlated detection of prompt neutrons using fast TOF}

The capacity to detect prompt fission neutrons in coincidence with prompt fission $\gamma$ rays opens up new possibilities to investigate as-yet unexplored correlations between neutrons and $\gamma$ rays. The $v$-Ball spectrometer has this capability due to the presence of the $\mathrm{LaBr}_{3}$ detectors in two rings at distances of $11 \mathrm{~cm}$ and $15 \mathrm{~cm}$ from the fission target. Since the neutron beam is pulsed with a period of $400 \mathrm{~ns}$ and a pulse width of $\sim 2 \mathrm{~ns}$, prompt neutrons can be detected via time-of-flight (TOF) from inelastic scattering in the $\mathrm{LaBr}_{3}$ detectors. Figure 4 shows the two dimensional separation of prompt $\gamma$ ray and prompt neutron events in the time-energy plane. Neutrons in the energy range $0.165-2.55 \mathrm{MeV}$ can be detected in the ring at a distance of $11 \mathrm{~cm}$ and $0.165-4.75 \mathrm{MeV}$ can be detected in the second ring at $15 \mathrm{~cm}$ corresponding to neutron flight times between 5 and $35 \mathrm{~ns}$. To be certain the delayed $\gamma$-ray detection originated from inelastic scattering of a prompt neutron in the detector an additional condition is placed on the $\gamma$ ray energy. Energies corresponding to the first or second excited states of ${ }^{139} \mathrm{La},{ }^{79,81} \mathrm{Br}$ must be detected at 165, 217, 261 and $276 \mathrm{keV}$ (see Fig. 4). The integral of spectral counts is proportional to the number of neutrons detected giving a total efficiency for neutron detection of $\bar{\varepsilon}=0.15 \%$. This efficiency is calibrated using the ${ }^{252} \mathrm{Cf}$ fission tagged data and the known ${ }^{252} \mathrm{Cf}$ neutron multiplicity of $\bar{v}=3.73$ per tagged fission event. A more sophisticated extension of the technique would be to weight each neutron according to the inelastic scattering cross section at the detected energy on an event-by-event basis.

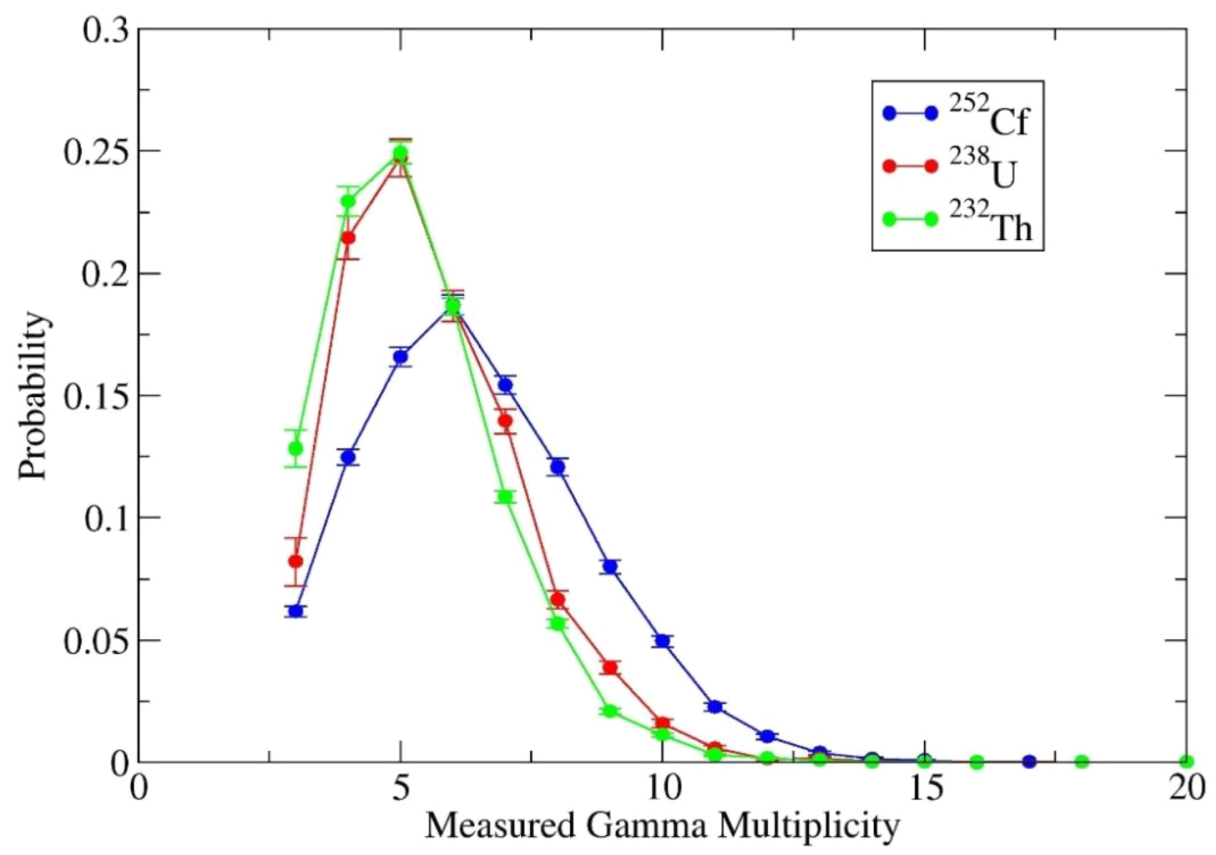

Figure 3. Comparison of the detected prompt $\gamma$-ray multiplicity distributions for the ${ }^{144} \mathrm{Ba}$ nucleus for the 3 different systems. Identification of ${ }^{144} \mathrm{Ba}$ is carried out by double-gating on the $4^{+} \longrightarrow 2^{+}$and $2^{+} \longrightarrow 0^{+}$transitions in the HPGe detectors.

For the neutron-induced fission data, care has to be taken to subtract off an appropriate background of neutrons uncorrelated in time due to scatterings occurring into the spectrometer from the neutron source itself. This problem does not occur for the ${ }^{252} \mathrm{Cf}$ spontaneous 
fission data. The capacity to detect prompt neutrons allows variations in prompt fission neutron multiplicity to be measured. Work to explore these neutron $/ \gamma$ correlations in fission is ongoing.

\section{Conclusions}

The $v$-Ball spectrometer coupled to the LICORNE neutron source offers some interesting new possibilities for deeper investigations into the fission process.
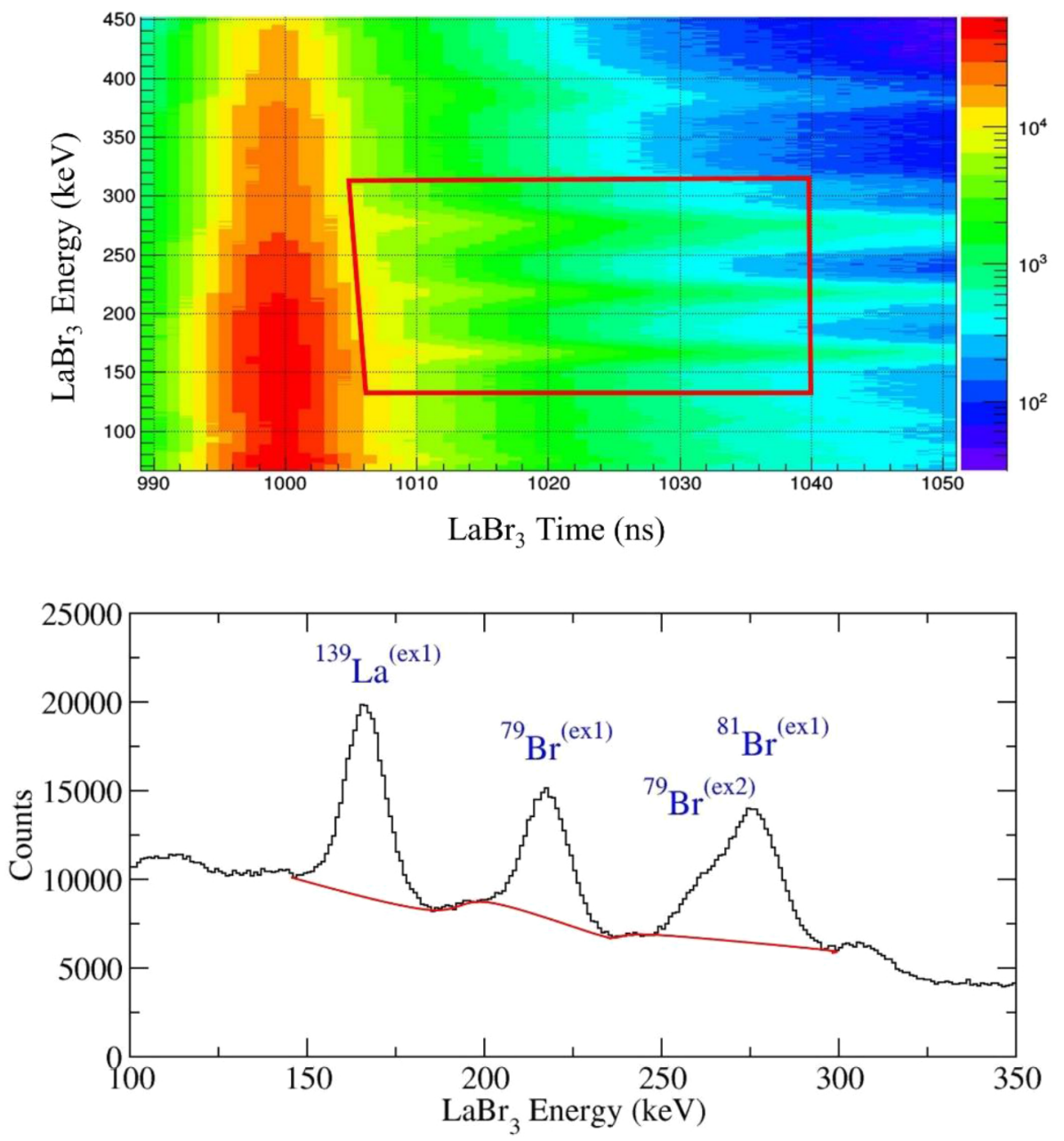

Figure 4. Detection of delayed $\gamma$ rays from inelastic scattering of prompt neutrons in the $\mathrm{LaBr}_{3}$ detectors. The top panel shows the 2-dimensional separation in the time-energy plane, while the bottom panel shows the projection onto the energy axis of the selected red zone and integration of the relevant peaks.

In particular, new observables such as prompt $\gamma$-ray multiplicity distributions are accessible in coincidence with particular fission fragments. Detection of prompt neutrons via timeof-flight over short distances is possible and variations in prompt neutron multiplicity can be 
measured. The complex correlations between these various observables can now be explored and analysis work is ongoing.

\section{References}

[1] M. Lebois, J.N. Wilson, et al., Nucl. Instrum. Meth. A 735, 145 (2014)

[2] J.N. Wilson, M. Lebois, M., et al., Phys. Proc. 64, 107 (2015)

[3] M. Lebois, N. Jovancevic, D. Thisse, R. Canavan, D. Étasse, M. Rudigier, J.N. Wilson, Nucl. Instrum. Meth. Phys. Res. A 960, 163580 (2020)

[4] J.N. Wilson et al., Phys. Rev. Lett. 118, 222501 (2017)

[5] H. Pasca et al., Phys. Rev. C 98, 014624 (2018)

[6] N. Fotiades et al., Phys. Rev. C 99, 024606 (2019)

[7] D. Ramos et al., Phys. Rev. Lett. 123, 092503 (2019)

[8] L. Gaudefroy, et al., Phys. Rev. C 97, 064317 (2018)

[9] J.N. Wilson et al., Nature 590, 566-570 (2021)

[10] M. Travar et al., Phys. Lett. B 817, 136293 (2021) 